\title{
Complicaciones del tratamiento con anticoagulantes orales en mayores de 65 años, a propósito de un caso
}

\author{
María de las Nieves Perejón Díaz ${ }^{1}$, María Jesús Rodríguez López², \\ Isabel Moreno Ruiz ${ }^{3}$ y Javier Benítez Rivero ${ }^{4}$ \\ ${ }^{1}$ Hospital Universitario Puerta del Mar (España); ${ }^{2}$ UGC Rodríguez Arias (España); \\ ${ }^{3}$ Centro de Salud Benalup Casas Viejas (España); ${ }^{4}$ UCG La Laguna (España)
}

Descripción del caso: Mujer de 79 años con antecedentes de hipertensión arterial, fibrilación auricular (FA) permanente anticoagulada (acenocumarol) e insuficiencia mitral. Barthel 90, Lowton-Brody: 2. Acude a urgencias por dolor costal (osteomuscular) de dos semanas de evolución, sin otros síntomas, siendo dada de alta y acudiendo nuevamente por epistaxis y disnea. Exploración y pruebas complementarias: destacar en la auscultación cardio-pulmonar: tonos arrítmicos con soplo sistólico en reborde esternal izquierdo, crepitantes hasta campos medios, con murmullo vesicular disminuido. Radiografía de tórax: masa hiliar derecha con engrosamiento pleural derecho, e infiltrado bilateral. Hemograma: hemoglobina $7.9 \mathrm{~g} / \mathrm{dl}$ (previa $11.6 \mathrm{~g} / \mathrm{dl}$ ), leucocitos 11.57x101. Bioquímica: PCR 72.90mg/l, urea $85 \mathrm{mg} / \mathrm{l}$. Coagulación: INR indeterminado, actividad protrombina $<12.5 \%$. Juicio Clínico: Hemorragia alveolar bilateral. Diagnóstico diferencial: Hemorragia alveolar bilateral, neumonía bilateral, insuficiencia cardiaca congestiva (Edema Agudo de Pulmón). Conclusión: El 0.5\% de la población usa anticoagulantes orales, entre el $6.8-11.1 \%$ por FA, un INR $>4$ aumenta el riesgo de sangrado. Se trata de un paciente pluripatológico, dependiente moderada, con mal cumplimiento tratamiento y de control de anticoagulación. Ante estos datos (anemización, ausencia de signos de infección o insuficiencia cardiaca), y los resultados de las pruebas, nos decantamos por la hemorragia alveolar como primera posibilidad diagnostica.

Palabras clave: Anticoagulantes orales, hemorragia alveolar, fibrilación auricular.

Complications of oral anticoagulant therapy in over 65 years, a case report. Case description: Woman, 79 years old. Medical history: hypertension, Atrial fibrillation (AF) permanent treated with acenocoumarol and mitral insufficiency. Barthel 90, Lowton-Brody. 2. The patient came to the emergency room for rib pain (musculoskeletal) in last two weeks, without other symptoms. We initiated analgesia and the patient was discharged. She came back with new symptoms: epistaxis and dyspnea. Exploration and complementary tests: cardio-pulmonary auscultation arrhythmic tones with sternal border systolic murmur left, crakling sounds in the lungs, with decreased breath sounds. Chest $\mathrm{x}$-ray: right hilar mass with right pleural thickening, and diffuse bilateral infiltration. CBC: hemoglobin $7.9 \mathrm{~g} / \mathrm{dl}$ (First: $11.6 \mathrm{~g} / \mathrm{dl}$ ), WBC $11.57 \times 101$. Biochemistry: PCR $72.90 \mathrm{mg} / \mathrm{l}$, urea $85 \mathrm{mg} / \mathrm{l}$. Coagulation: INR undetermined, activity prothrombin $<12.5 \%$. Diagnosis: bilateral alveolar hemorrhage. Diagnosis Differential: bilateral alveolar hemorrhage, bilateral pneumonia, heart failure congestive (Acute Lung Edema). Conclusion: $0.5 \%$ of the population uses oral anticoagulants, between $6.8-11.1 \%$ by FA an INR>, increases the risk of bleeding. This is a patient with multimorbidity, moderately dependent, with poor treatment compliance and anticoagulation control. Given these data (anemia, no signs of infection or heart failure), and the test results, we opted for the first possibility alveolar hemorrhage diagnosed. Keywords: Oral anticoagulants, alveolar hemorrhage, atrial fibrilation.

Correspondencia: María de las Nieves Perejón Díaz. Servicio Cuidados Críticos y Urgencias, Hospital Universitario Puerta del Mar. C/ General García de la Herrán, 34, 5º, San Fernando. C.P.: 11.100. Cádiz (España). E-mail: nieves.perejondiaz@gmail.com 


\section{Descripción del caso}

Nos encontramos ante una mujer de 79 años, que acude a urgencias por dolor costal derecho de características mecánicas, aunque sin referir sobreesfuerzos ni traumatismos previos, así mismo presenta mal estar general, tos seca, y ha tenido sensación de distermia, pero no se ha termometrado fiebre. Entre sus antecedentes personales cabe destacar:

- Antecedentes cardiológicos: hipertensión arterial de larga evolución, miocardiopatia hipertrófica septal no obstructiva, fibrilación auricular (FA) crónica anticoagulada (acenocumarol) e insuficiencia mitral leve.

- Síndrome depresivo mayor.

- Osteoatrosis generalizada.

- Intervenciones quirúrgicas: Colecistectomía, apendicetomía.

- Tratamiento habitual: sertralina $100 \mathrm{mg} \mathrm{1/24}$ horas, furosemida $40 \mathrm{mg} \mathrm{1/24}$ horas, espironolactona $25 \mathrm{mg} 1 / 24$ horas, diltiazem $120 \mathrm{mg} 1 / 12$ horas, acenocumarol $4 \mathrm{mg}$ según pauta establecida en control de anticoagulación en su centro de salud.

En la evaluación geriátrica inicial presentaba un test de Barthel 90 (Dependiente moderado) y Lawton-Brody: 2 (Dependiente severa). Una vez evaluada la paciente y apoyándonos en los estudios complementarios, es dada de alta derivándose a neumología para estudio de probable masa hiliar derecha y engrosamiento pleural de forma ambulatoria. Unos días después la paciente acude nuevamente por cuadro de mal estar general, con disnea, empeoramiento de la tos y epistaxis de repetición desde esa tarde. Continua sin fiebre, y el dolor costal ha respondido bien a la analgesia.

La familia refiere además que la paciente estaba pendiente por su médico de completar estudio de deterioro cognitivo, desde hace meses no realiza correctamente su tratamiento, y no acude a los controles de anticoagulación.

\section{Exploración y pruebas complementarias}

En la primera asistencia en urgencias la exploración física fue la siguiente: Buen estado general, palidez mucocutanea. No ingurgitación yugular, ni adenopatías laterocervicales. Auscultación cardio-pulmonar: tonos arrítmicos con soplo sistólico en reborde esternal izquierdo II/VI, con murmullo vesicular disminuido crepitantes en ambas bases pulmonares. Dolor a la palpación arcos costales inferiores derechos. Abdomen blando y depresible, no doloroso a la palpación, sin masas ni visceromegalias, ni datos de irritación peritoneal, ruidos hidroaereos presentas, y puño-percusión renal bilateral, negativa. A nivel de miembros inferiores, pulsos distales simétricos y conservados, con datos de insuficiencia venosa crónica, no edemas, no signos de trombosis venosa profunda ni superficial. Exploraciónn neurológica normal. Al acudir al día siguiente la exploración en similar, salvo la auscultación pulmonar, donde ya se 
apreciaban crepitantes bilaterales hasta campos medios. A la llegada se toman constantes vitales, presentadas en la siguiente tabla.

Tabla 1. Constantes vitales

\begin{tabular}{lcc}
\hline \multicolumn{1}{c}{ Constantes } & Primera asistencia & Segunda asistencia \\
\hline Presión arterial & $109 / 60 \mathrm{mmHg}$ & $100 / 65 \mathrm{mmHg}$ \\
\hline Saturación oxígeno & $97 \%$ & $92 \%$ \\
\hline Frecuencia cardiaca & $74 \mathrm{lpm}$ & $98 \mathrm{lpm}$ \\
\hline Temperatura & $36^{\circ}$ & $36^{\circ}$ \\
\hline
\end{tabular}

En cuanto a las exploraciones complementarias, a continuación detallaremos los resultados y las diferencias encontrados, entre las realizadas en la primera vez que la paciente acude a urgencias (Analítica con hemograma y coagulación, radiografía de tórax y electrocardiograma), y las realizadas en una segunda instancia (Se repitieron las mismas pruebas complementarias, y se añadió un estudio básico de la coagulación).

Radiografía de tórax:

$1^{\circ}$ Radiografía de tórax: se informad como probable masa hiliar derecha, con pinzamiento seno costo-frénico derecho y engrosamiento pleural de ese mismo lado. Aumento de la trama bronquialveolar. Cardiomegalia.
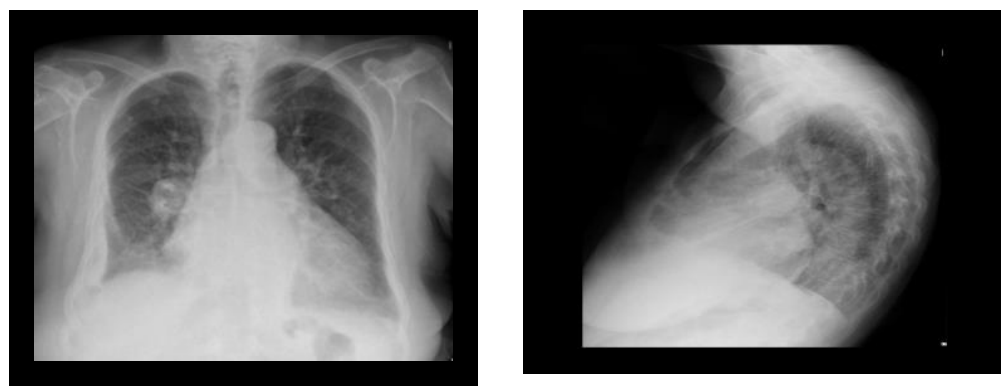

$2^{\circ}$ Radiografía de tórax: infiltrado intersticial bilateral, con pinzamiento seno costo-frénico derecho y engrosamiento pleural de ese mismo lado. Cardiomegalia.
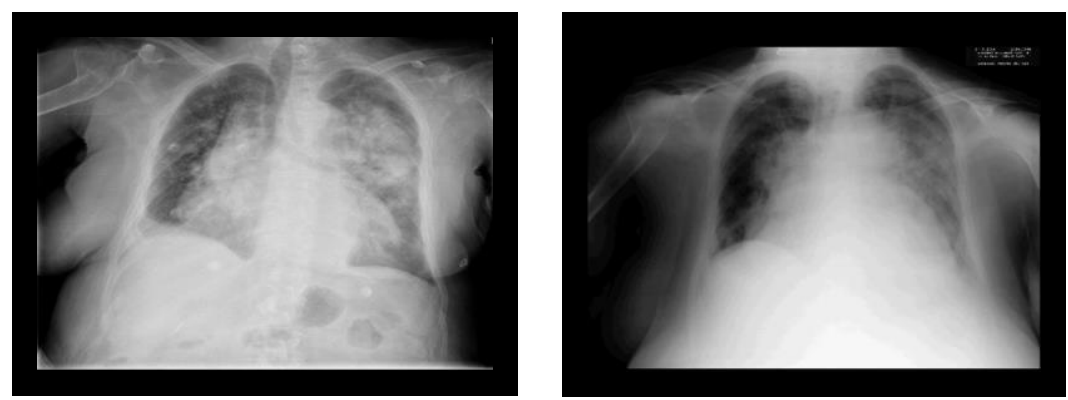
A la semana del ingreso, se realiza un nuevo control radiológico por persistencia de la disnea. En la primera evaluación en urgencias se solicita hemograma y bioquímica, pero no coagulación. Los resultados se exponen en la siguiente tabla, comprados con los de la segunda asistencia, donde tras ver las imágenes radiológicas, se solicita estudio básico de coagulación.

Tabla 2. Analítica: hemograma

\begin{tabular}{lcc}
\hline HEMOGRAMA & Primera asistencia & Segunda asistencia \\
\hline Hemoglobina & $11.6 \mathrm{~g} / \mathrm{dl}$ & $7.9 \mathrm{~g} / \mathrm{dl}$ \\
\hline Hematocrito & $35 \%$ & $23 \%$ \\
\hline Hematíes & $4.12 \mathrm{millón} /$ & $2.72 \mathrm{millón} /$ \\
\hline Leucocitos & $5.82 \times 10^{3} /$ & $7.20 \times 10^{3} /$ \\
\hline Neutrofilos & $4.85 \times 10^{3} /$ & $5.98 \times 10^{3} /$ \\
\hline Plaquetas & $150 \times 10^{3} /$ & $173 \times 10^{3} /$ \\
\hline
\end{tabular}

Tabla 3. Analítica: bioquímica

\begin{tabular}{lcc}
\hline \multicolumn{1}{c}{ BIOQUIMICA } & Primera asistencia & Segunda asistencia \\
\hline Glucemia & $123 \mathrm{mg} / \mathrm{dl}$ & $95 \mathrm{mg} / \mathrm{dl}$ \\
\hline Urea & $85 \mathrm{mg} / \mathrm{dl}$ & $126 \mathrm{mg} / \mathrm{dl}$ \\
\hline Creatinina & $1.2 \mathrm{mg} / \mathrm{dl}$ & $1.8 \mathrm{mg} / \mathrm{dl}$ \\
\hline Sodio & $126 \mathrm{mmol} / \mathrm{L}$ & $120 \mathrm{mmol} / \mathrm{L}$ \\
\hline Potasio & $4.49 \mathrm{mmol} / \mathrm{L}$ & $5.24 \mathrm{mmol} / \mathrm{L}$ \\
\hline Proteína C Reactiva & $72.90 \mathrm{mg} / \mathrm{l}$ & $50.98 \mathrm{mg} / \mathrm{l}$ \\
\hline
\end{tabular}

Tabla 4. Estudio básico de coagulación

\begin{tabular}{lcc}
\hline \multicolumn{1}{c}{ Coagulación } & Primera asistencia & Segunda asistencia \\
\hline \multicolumn{1}{c}{ INR } & NO SE REALIZA ESTUDIO & NO CALCULADO \\
\hline Actividad de protrombina & $<12.5 \%$ \\
\hline AP- Mezcla normal & $68 \%$ \\
\hline Tiempo de cefalina (TPTA) & 150 segundos \\
\hline TPTA mezcla normal & 36.80 segundos \\
\hline Fibrinogeno & $376 \mathrm{mg} / \mathrm{dl}$ \\
\hline
\end{tabular}

Se administra vitamina $\mathrm{K}$ en urgencias, y se solicita nueva analítica:

Tabla 5. Analítica a (hemograma y bioquímica) de control tras tratamiento

\begin{tabular}{lclc}
\hline \multicolumn{2}{c}{ HEMOGRAMA } & \multicolumn{2}{c}{ Bioquímica } \\
\hline Hemoglobina & $6.4 \mathrm{~g} / \mathrm{dl}$ & Glucemia & $78 \mathrm{mg} / \mathrm{dl}$ \\
\hline Hematocrito & $19 \%$ & Urea & $140 \mathrm{mg} / \mathrm{dl}$ \\
\hline Hematíes & $2.30 \mathrm{millón} /$ & Creatinina & $1.7 \mathrm{mg} / \mathrm{dl}$ \\
\hline Leucocitos & $5.35 \times 10^{3} /$ & Sodio & $126 \mathrm{mmol} / \mathrm{L}$ \\
\hline Neutrofilos & $3.94 \times 10^{3} /$ & Potasio & $4.59 \mathrm{mmol} / \mathrm{L}$ \\
\hline Plaquetas & $156 \times 10^{3} /$ & Proteína C Reactiva & $49.20 .90 \mathrm{mg} / \mathrm{l}$ \\
\hline
\end{tabular}


Tabla 6. Estudio básico de coagulación de control tras tratamiento

\begin{tabular}{|c|c|}
\hline \multicolumn{2}{|c|}{ Coagulación } \\
\hline INR & 1.5 \\
\hline Actividad de protrombina & $58 \%$ \\
\hline AP- Mezcla normal & $90 \%$ \\
\hline Tiempo de cefalina (TPTA) & 39 segundos \\
\hline TPTA mezcla normal & 34.60 segundos \\
\hline Fibrinogeno & $332 \mathrm{mg} / \mathrm{dl}$ \\
\hline
\end{tabular}

Tabla 7. Electrocardiograma

\begin{tabular}{lcc}
\hline & Primera asistencia & Segunda asistencia \\
\hline \multirow{3}{*}{ ELECTROCARDIOGRAMA } & Fibrilación auricular a 80 sístoles & Fibrilación auricular a 95-100 \\
& eléctricos por minuto, sin & sístoles eléctricos por minuto, sin \\
& alteraciones agudas de la & alteraciones agudas de la \\
& repolarización. No bloqueos & repolarización. No bloqueos \\
\hline
\end{tabular}

\section{Juicio Clínico}

Con los antecedentes de la paciente y los resultados de los estudios complementarios realizados, se llega al diagnóstico de: Hemorragia alveolar bilateral, resultado de una complicación secundaría al tratamiento oral con acenocumarol.

\section{Diagnóstico diferencial}

Dentro del diagnóstico diferencial se plantearos tres entidades como causantes de cuadro de la paciente: hemorragia alveolar bilateral, neumonía bilateral, insuficiencia cardiaca congestiva (Edema Agudo de Pulmón).

Hemorragia alveolar bilateral: Los síntomas más comunes de la HAD son hemoptisis, disnea y tos de inicio agudo o subagudo. La exploración física generalmente es inespecífica. En los casos secundarios a una enfermedad sistémica puede haber signos clínicos relacionados con las enfermedades de base. En los estudios de laboratorio se evidencia caída de hemoglobina y hematocrito (Buendía-Roldán, 2009).

Neumonía bilateral: es importante realizar una historia clínica detallada dirigida a buscar síntomas de infección, así como del entorno en el que se puede producir la infección y las posibles exposiciones específicas (contacto con animales, viajes, aerosoles, zonas endémicas). Los síntomas más frecuentes son tos, fiebre, expectoración mucopurulenta, dolor torácico de tipo pleurítico y malestar general. En los pacientes ancianos los síntomas suelen ser inespecíficos, muchas veces sin fiebre y con cuadros de deterioro del estado general o neurológicos como confusión (Garcia, 2011).

Insuficiencia Cardiaca Descompensada (Edema Agudo de Pulmón): según la última. Guía de práctica clínica de la Sociedad Europea de Cardiología (ESC) para el diagnóstico y tratamiento de la insuficiencia cardiaca (Dickstein, 2008), la insuficiencia cardiaca es un síndrome clínico en el que los pacientes presentan las siguientes características: 
- Síntomas típicos de insuficiencia cardiaca (falta de aire en reposo o durante el ejercicio, fatiga, cansancio, inflamación de tobillos).

- Signos típicos de insuficiencia cardiaca (taquicardia, taquipnea, estertores pulmonares, derrame pleural, elevación de la presión yugular venosa, edema periférico, hepatomegalia).

- Evidencia objetiva de una anomalía estructural o funcional del corazón en reposo (cardiomegalia, tercer sonido, soplos cardiacos, anomalías electrocardiográficas, concentraciones elevadas de péptidos natriuréticos).

\section{CONCLUSIÓN}

Nos encontramos ante un caso de compilación mayor del tratamiento con anticoagulantes orales, que son usados en un $0.5 \%$ de la población (Birrium, 2001), entre el 6.8-11.1\% por FA (Fernández, 1999), un INR $>4$ aumenta el riesgo de sangrado (Diez, 2013).

La fibrilación auricular (FA) es la arritmia cardiaca sostenida más frecuente en la población, en torno a un 1-2\% del total, porcentaje que se espera que se duplique en los próximos 50 años, en los mayores de 80 años se sitúa ya en el 5-15\% (Camm, 2011).

En todo paciente con FA se debe establecer una estratificación del riesgo accidente cerebrovascular (ACV) y de tromboembolia. A día de hoy se utiliza el índice de CHADS2 (insuficiencia cardiaca, hipertensión, edad, diabetes mellitus, ACV).

A día de hoy los fármacos más utilizados son los antagonistas de la vitamina $\mathrm{K}$, requieren de una monitorización continua y no están exentos de producir complicaciones hemorrágicas graves, de ahí su infrautilización, sobretodo en pacientes mayores de 65 años.

Las hemorragias graves constituyen la complicación más importante de este tratamiento, y se definen por su localización, por la necesidad de transfusión, o la amenaza que supone para la vida del paciente.

Hay una serie de factores que incrementan el riesgo de su aparición (Martí, 2014):

- Edad avanzada (sobretodo > de 75 años).

- Hipertensión arterial no controlada

- Abuso de alcohol

- Hepatopatías

- Antecedentes hemorrágicos previos

- Uso concomitante de antiagregantes

- Fibrilación auricular 
- Insuficiencia Renal

- $\quad$ INR $>3$

- Anemia

La frecuencia de las hemorragias graves va del 1.2 al 7.1\%. Las hemorragias mortales van del $0.4-8 \%$.

Si nos encontramos ante una serie de fármacos de difícil manejo en la población en general, además hay que tener en cuenta que los pacientes mayores de 65 años en lo referente a la adherencia terapéutica la complejidad esta aumentada por varios factores: polimedicación y mayor probabilidad de aparición de reacciones adversas medicamentosas, morbilidad, mayor número de interacciones farmacológicas y el deterioro cognitivo (Sáez de la Fuente, 2004).

En este caso se trata de un paciente pluripatológico (Fernández, 1999), en la que además se dan una serie de factores que favorecen la aparición de complicaciones relacionadas con la toma de acenocumarol vía oral, ya que es un tratamiento que como hemos mencionado con anterioridad requiere un exhaustivo seguimiento. Del mismo modo se trata de una paciente polimedicada, y además con un deterioro cognitivo establecido, y que no cuenta con un soporte familiar adecuado.

A día de hoy contamos con nuevos fármacos como los inhibidores orales del factor Xa, entre ellos el rivaroxabán y apixabán, o el inhibidor oral de la trombina, dabigatrán. Hay una serie de ventajas que creemos importante señalar (Mateo, 2013):

- Rápido comienzo de acción

- No es necesaria terapia puente

- Efecto anticoagulante predecible

- No es necesaria monitorización rutinaria

- Objetivo terapéutico específico

- $\quad$ Bajo riesgo de efectos adversos

- Pocas interacciones con alimentos

- No implican limitaciones dietéticas

- Pocas interacciones con fármacos

- Pocas restricciones de fármacos

A día de hoy hay dos grandes indicaciones para el uso de nuevos anticoagulantes: prevención primaria de episodios tromboembólicos venosos en pacientes adultos sometidos a cirugía de reemplazo total de cadera o cirugía de reemplazo de rodilla, programadas y prevención del ictus y de la embolia sistémica en pacientes adultos con fibrilación auricular no valvular.

El empleo de esta nueva línea farmacológica, además de mejorar la calidad de vida de nuestros pacientes, disminuiría el número de complicaciones graves, siempre y cuando estos pertenezcan uno de los grupos para los que a día de hoy están indicados. 


\section{REFERENCIAS}

Biurrun, L.M., Esteban, M.S., y Díaz, A. (2001). Manejo de anticoagulantes orales en atención primaria. SEMERGEN; 27, 301-312.

Ludlam, C.A. Bennett, B., Fox, K.A., Lowe, G.D. y Reid, A.W. (1995). Guidelines on Oral anticoagulation: Haemostasis and Thrombosis Task Force for the Bristish Commitee for Standars in Haematology. Blood Coagul Fibrinolysis, 6(3), 273-285.

Camm, A.J., Kirchhof, P., Y.H. Lip, G., Schotten, U., Savelieva, I., Ernst, S., Van Gelder,I., AlAttar, N., Hindricks, G., Prendergast, B., Heidbuchel, H., Alfieri, O., Angelini, A., Atar, D., Colonna, P., De Caterina, R., De Sutter, J., Goette, A., Gorenek, B., Heldal, M., Hohloser, S., Kolh, P., Le Heuzey, J., Ponikowski, P. y Rutten, F. (2011). Guías de práctica clínica para el manejo de la fibrilación auricular. Revista Española de Cardiología; 63, 14-83.

Díez-Manglano, J., Bernabeu-Wittel, M., Barón-Franco, B., Murcia-Zaragoza, J., Fuertes, A., Alemán, A. y Ollero-Baturone, M. (2013). Anticoagulación en pacientes pluripatológicos con fibrilación auricular. Medicina Clínica, 140, 97-103.

Fernández, M.A. (1999). El paciente anticoagulado y el médico de cabecera. Barcelona: Laboratorios Esteve.

Escolar, G., García, J., López, M.F. y Roldán, V. (2012). Guía sobre los nuevos anticoagulantes orales. Murcia: Sociedad Española de Hematología y Hemoterapia.

Marti-Fabregas, J., Delgado-Mederos, D. y Mateo, J. (2012). Limitaciones del tratamiento anticoagulante. Neurología, 27, 27-32.

Mateo, J. (2013). Nuevos anticoagulantes orales y su papel en la práctica clínica. Revista Española de Cardiología, 13, 33-41.

Nuevo, J.A., Cano, J.C., Pintor, E., Braun, B., Visús, E. y Sevillano, J.A. (2008). Exceso de anticoagulación oral: análisis desde un servicio de Urgencias. Revista Clinica Española, 208(2), 66-70.

Petal, A., Wijnen, P.A., Linssen, C.F., Haenen, G.R., Bekers, O. y Drent, M. (2010). Variant VKORC1 and CYP"C) Alleles in Patient with Difuse Alveolar Hemorrhage Caused by Oral Anticoagulants. Molecular and Diagnosis and Therapy, 14, 23-30.

Polo, J. (2013). Nuevos anticoagulantes frente a anticoagulantes clásicos: ventajas e inconvenientes. SEMERGEN, 39, 10-16.

Sáez de la Fuente, J., Granja, V., Lechuga, P., Otero, B., Herreros, A. y Medina, J. (2011). Eficacia de la información al alta en la adherencia del paciente polimedicado. Farmacología Hospitalaria, 35, 128-134.

Sánchez, J., Guidín-Uriel, M. y Ridocci-Soriano, F. (2006). Seguridad de la asociación de aspirina, clopidogrel y acenocumarol en pacientes con indicación de anticoagulación. Revista Española de Cardiología, 59, 1345-1346.

Recibido: 20 abril de 2014

Recepción Modificaciones: 26 de mayo de 2014

Aceptado: 2 de junio de 2014 